\title{
RECURSIVE FUNCTIONS OVER WELL ORDERED PARTIAL ORDERINGS ${ }^{1}$
}

\author{
HARTLEY ROGERS, JR.
}

One of the basic tools of research in recursive function theory is the Recursion Theorem. Statement and detailed proof of this theorem appear in [1]. As formulated for functions of one variable, the recursion theorem asserts, first, that for any recursive function $h$, there exists an integer $x_{0}$ such that $\phi_{x_{0}}$ and $\phi_{h\left(x_{0}\right)}$ are identical as partial functions; i.e. each represents, extensionally, the same map from the same subset of the non-negative integers into the non-negative integers. (Notation: $N$ is the set of all non-negative integers. $a, b, \cdots$, $x, y, \cdots$ are members of $N . \phi_{x}, x=0,1,2, \cdots$ are the partial recursive functions under some standard Gödel numbering. $f, g, h, \cdots$ are functions mapping $N$ into $N$. If $\psi$ and $\phi$ are partial functions, $\psi=\phi$ means that $\psi$ and $\phi$ are the same map, defined on the same subset of $N$, into $N . \psi=\phi$ on $D$, where $D \subset N$, means that, when restricted to $D, \psi$ and $\phi$ are the same map, defined on the same subset of $D$, into $N$. Henceforth, "integer" shall mean member of $N$ ). The recursion theorem asserts, second, that there is a uniform effective method for computing $x_{0}$ from a Gödel number for the given $h$. As has been remarked, the theorem is essentially a fixed point theorem. In general it is used to show the existence of functions that are implicitly defined in certain stipulated ways. In this respect its use is similar to the use of the more classical fixed point theorems of topology and analysis.

In outline, the proof of the theorem is quite simple. Let $x$ be given. Consider the partial recursive function $\psi$ defined as follows.

$$
\psi(z) \simeq\left\{\begin{array}{l}
\phi_{\phi_{x}(x)}(z), \text { if } \phi_{x}(x) \text { is defined } \\
\text { undefined, if } \phi_{x}(x) \text { is undefined }
\end{array}\right.
$$

(Notation: $\psi(z) \simeq \phi(z)$, for partial recursive functions $\psi$ and $\phi$, means that $\psi=\phi$; see $[1$, p. 327]). This definition itself provides a uniform method for computing a Gödel number for $\psi$ from $x$. That is to say, there is a recursive function $g$ such that

Received by the editors October 4, 1958 and, in revised form, January 26, 1959.

${ }^{1}$ Results of the kind given in this paper were first summarized in an abstract, J. Symb. Logic vol. 23 (1958). Dr. D. L. Kreider pointed out to the author that the lemma stated there, while true, is not sufficiently general for the intended applications. This defect is corrected in the present paper. 


$$
\phi_{\theta(x)}(z) \simeq \begin{cases}\phi_{\phi_{x}(x)}(z), & \text { if } \phi_{x}(x) \text { is defined } \\ \text { undefined, } & \text { if } \phi_{x}(x) \text { is undefined }\end{cases}
$$

Now let $h$ be given and let $y_{0}$ be a Gödel number for $h \circ g$. Then we have $\phi_{g\left(y_{0}\right)}=\phi_{\phi_{y_{0}}\left(y_{0}\right)}=\phi_{h\left(g\left(y_{0}\right)\right)}$ and $x_{0}=g\left(y_{0}\right)$ is the desired fixed point value. Clearly $x_{0}$ is computable in a uniform effective manner from a Gödel number for $h$.

Applications of this theorem have been of two general kinds. The first kind concern the theory of recursively invariant properties of sets of integers. Here, the recursion theorem is usually used in the weaker form: for any recursive $h$, there is an $x_{0}$ such that $W_{x_{0}}=W_{h\left(x_{0}\right)}$ (where $W_{x}$ is the range of $\phi_{x}, x=0,1,2, \cdots$ ). An example of such use is Myhill's proof of the recursive isomorphism of creative sets, [2]. The second kind of application occurs in the theories of constructive ordinal notations and predicate hierarchies. Examples of such use are found in $[3 ; 4 ; 5]$. We shall be henceforth concerned with applications of this second kind.

These applications have certain common features which we formulate as a general lemma and give in the present paper. The common features are not immediately evident in the various occurrences in the literature. An explicit formulation of them is of value, both as an intuitive guide to the beginner and as a heuristic aid in research. Some of the applications concern the existence of certain partial recursive functions defined over the well ordered partially ordered tree $\left\langle O,<_{0}\right\rangle$ of constructive ordinal notations (see [3]); roughly speaking, these applications can be summarized in the following principle: if, from any partial recursive function defined on a segment of $<_{0}$ and possessing certain specified desirable properties, one can effectively find a partial recursive extension of that function defined on an extension of that segment and possessing those properties, then there is a partial recursive function defined on $<_{0}$ possessing those properties. Other applications concern the existence of functions related to $<_{o}$ in a somewhat more complex way. In our lemma and the illustrations which follow it, we shall show that all of these applications can be embodied in a certain recursive principle of transfinite induction. This principle is similar to that stated above for $<_{o}$, but with the provision that $<_{0}$ is replaced by any well ordered partial ordering of integers. Thus the special recursive structure of $<_{o}$ plays a less important role than might have been suspected.

Definitions. The relation $<_{W}$ defined on a certain set $W$ of integers will be called a partial ordering if it is transitive and irreflexive. From now on we assume that $<_{W}$ is such a relation. 
For such a relation, $a \leqq_{W} b$ shall mean $\left[a<_{W} b\right.$ or $\left.a=b\right]$.

$<_{W}$ will be said to satisfy the descending chain condition if there is no infinite sequence $\left\{a_{i}\right\}$ of elements of $W$ such that $a_{j}<_{W} a_{i}$ for all $i, j$ such that $i<j$.

An element $m$ of $W$ will be called minimal in the set $D$ if $m \in D$ and there is no $a \in D$ such that $a<_{W} m$. We say that $<_{W}$ is well ordered if every nonempty subset $D$ of $W$ contains an element which is minimal in $D$.

It is easy to show that $<_{W}$ satisfies the descending chain condition if and only if $<_{W}$ is well ordered. (The Axiom of Choice is not necessary for this proof since $W$ is a set of non-negative integers, and the natural ordering of the integers provides us with a choice principle for all occasions.)

A relation will be called well founded, if it is a well ordered partial ordering. This terminology derives from [7] where such relations are studied in a general set theoretic context.

$S$ is a segment of $W$ if $S \subset W$ and if $\left[\left[a \in S\right.\right.$ and $\left.\left.b<_{W} a\right] \Rightarrow b \in S\right]$. For $a \in W,(a)$ shall be the segment $\left\{b \mid b<_{W} a\right\}$; and $(a]$ shall be the segment $\left\{b \mid b \leqq_{W} a\right\}$. Note that $W$ is a segment of itself.

Lemma. Let $<_{W}$ be a well founded relation on $W$. Let $D(x, S)$ be a relation between integers $x$ and segments $S$ of $W$ such that

$$
\begin{aligned}
{\left[D(x, S) \text { and } \phi_{x}=\phi_{y} \text { on } S\right] \Rightarrow } & \mathscr{D}(y, S), \\
& \text { ("extensionality" assumption); }
\end{aligned}
$$

Let $f$ be a recursive function of two variables such that

$$
\begin{array}{r}
D(x,(a)) \Rightarrow\left[D(f(x, a),(a]) \text { and } \phi_{f(x, a)}=\phi_{x} \text { on }(a)\right], \\
\text { ("effectiveness" assumption). }
\end{array}
$$

Then there exists an integer $x_{0}$ such that $D\left(x_{0}, W\right)$.

Before giving the proof, we comment that the relation $D$ represents some given criterion of desirability for partial recursive function $\phi_{x}$ defined on segment $S$. For the lemma to apply, this criterion must satisfy conditions (i) and (ii). The function $f$ represents an effective procedure for extending any "desirable" partial recursive function on a given segment to a "desirable" partial recursive function on the larger segment. The lemma then asserts that, in the presence of (i), (ii) and (iii), a "desirable" partial recursive function exists on $W$. For example, $<_{W}$ might be $<_{0}$ and $\mathfrak{D}(x, S)$ might assert: $\phi_{x}$ is defined on $S$ and for any $a \in S, \phi_{x}(a)$ gives, as value, $2^{p} 3^{q}$ where $p$ is a Gödel num- 
ber for the predicate $H_{a}$ in one-function-quantifier-existential form and $q$ is a Gödel number for $H_{a}$ in one-function-quantifier-universal form. (For notation and terminology, see [4] or [6].) In this case $f$ would embody the intuitively rather simple inductive procedure for getting the one-function-quantifier forms for $H_{a}$ from those for $H_{b}$, $b<. a$. Conditions (i), (ii) and (iii) hold, and application of the lemma gives the existence of a recursive function, defined over the whole of $<_{0}$, with the desired properties. This result is Theorem 9 of [4] (cf. Theorem XXI of [6]).

Comment. The reader will observe suggestive similarities between the lemma and some of the inductive principles of classical set theory by which continuous ordinal-valued functions may be defined over segments of the ordinal numbers. The special feature of the lemma is, of course, that the ultimate function produced is partial recursive.

Proof of the Lemma. Consider the partial recursive function $\psi$ defined by: $\psi(a) \simeq \phi_{f(x, a)}(a)$. A recursive function $h$ can be obtained giving a Gödel number for $\psi$ as a function of $x$. Applying the recursion theorem, we get an $x_{0}$ such that

$$
\phi_{x_{0}}(a) \simeq \phi_{f\left(x_{0}, a\right)}(a) \text { as } a \text { varies. }
$$

We now show, by contradiction, that $D\left(x_{0}, W\right)$. Assume not $\mathscr{D}\left(x_{0}, W\right)$. Then by (ii), not $D\left(x_{0},(a]\right)$ for some $a \in W$; i.e., $\{a \mid a \in W$ and not $\left.D\left(x_{0},(a]\right)\right\}$ is nonempty. Choose a minimal member $m$ of this set. Then $D\left(x_{0},(b]\right)$ for all $b<_{w} m$, and by (ii), $D\left(x_{0},(m)\right)$. Hence, by (iii), $D\left(f\left(x_{0}, m\right),(m]\right)$ and $\phi_{f\left(x_{0}, m\right)}=\phi_{x_{0}}$ on $(m)$. But now, by (iv), $\phi_{x_{0}}=\phi_{f\left(x_{0}, m\right)}$ on $(m]$, and hence by (i), $\mathfrak{D}\left(x_{0},(m]\right)$; a contradiction. This completes the proof.

Two plausible alterations of the lemma suggest themselves. They are: $(\alpha)$ try to drop the " $\phi_{f(x, a)}=\phi_{x}$ on $S_{a}$ " condition in (iii); and $(\beta)$ try to obtain the converse of (ii) as an additional conclusion. Neither of these is successful. In case $(\alpha)$, let $W$ be the integers in natural order, and let $D(x, S)$ mean: $\phi_{x}$ is defined on $S$ and is monotone decreasing on $S$. Then (i), (ii) and the modified (iii) would hold; but $D(x, W)$ is false for any $x$. In case $(\beta)$, let $W$ be the integers in natural order; let $D(x, S)$ be true for any $x$ if $S$ is empty; let $D(x,(b])$ be true if and only if $\phi_{x}(b)$ is defined and $\phi_{x}(b) \geqq b$; and let $D(x, W)$ be true if and only if $\phi_{x}$ has infinite range. Then both assumptions and conclusion of the lemma hold, but the converse of (ii) does not hold for all $x$ and $S$.

Parameters. Parameters can be given a convenient and straightforward role in the recursion theorem. Let $p$ be the integer-valued parameters $p_{1}, p_{2}, \cdots, p_{k}$. We say that the function $h^{(p)}$ is a recur- 
sive function of $l$ variables effective in $p$, if there is a recursive function $f$ of $k+l$ variables such that $h^{(p)}(\mathbf{x}) \simeq f(\mathbf{x}, \boldsymbol{p})$ (where $\mathrm{x}$ represents the variables $\left.x_{1}, x_{2}, \cdots, x_{l}\right)$; this is in turn equivalent to the statement that there is a recursive function $f^{\prime}$ of $k$ variables such that $h^{(p)}(\mathbf{x})$ $\simeq \phi_{f^{\prime}(p)}(x)$. The initial formulation of the recursion theorem can now be given in a stronger parametric form: for any recursive function $h^{(p)}$ of one variable, effective in $p$, there is an $x_{0}$ such that $\phi_{x_{0}(p)}$ $=\phi_{h}(p) x_{\left(x_{0}(p)\right)}$ where $x_{0}(p)$ is a recursive function of $k$ variables; furthermore there is a uniform effective procedure for getting a Gödel number for the function $x_{0}$ from a Gödel number for the function of $k+1$ variables denoted as $h^{(p)}$. The proof follows by trivial modification of the initial proof for the recursion theorem outlined above.

A corresponding strengthened form of our lemma can also be given. We introduce parameters $\boldsymbol{p}$ and let $W, \mathfrak{D}$, and $f$ all depend upon $\boldsymbol{p}$. Only the dependence of $f$ on $\boldsymbol{p}$ need be effective. We have:

Let $<_{W}(p)$ be a well founded relation on $W^{(p)}$. Let $(a)^{(p)}$ $=\left\{b \mid b<_{W}^{(p)} a\right\}$ and $(a]^{(p)}=\left\{b \mid b \leqq W^{(p)} a\right\}$. Let $\mathscr{D}^{(p)}(x, S)$ be a relation between integers $x$ and segments $S$ of $W^{(p)}$ satisfying the conditions

$$
\begin{aligned}
& {\left[D^{(p)}(x, S) \text { and } \phi_{x}=\phi_{\nu} \text { on } S\right] \Rightarrow D^{(p)}(y, S),} \\
& D^{(p)}\left(x,(a]^{(p)}\right) \text { for all } a \in S \Rightarrow D^{(p)}(x, S) .
\end{aligned}
$$

Let $f^{(p)}$ be a recursive function of two variables effective in $p$ such that

$$
\begin{aligned}
\mathfrak{D}^{(p)}\left(x,(a)^{(p)}\right) & \Rightarrow\left[D^{(p)}\left(f^{(p)}(x, a),(a]^{(p)}\right)\right. \text { and } \\
\phi_{f}(p)(x, a) & \left.=\phi_{x} \text { on }(a)^{(p)}\right] .
\end{aligned}
$$

Then there exists $x_{0}$, a recursive function of $k$ variables, such that for all $\boldsymbol{p}, \mathfrak{D}^{(p)}\left(x_{0}(\boldsymbol{p}), W^{(p)}\right)$.

The proof is the same as before, except that $x_{0}$ is obtained by an application of the recursion theorem to yield $\phi_{x_{0}}(a) \simeq \phi_{f}(p){ }_{\left(x_{0}, a\right)}(a)$ as $a$ varies. In many applications it will be true that for any $x, \phi_{f}(p)(x, a)(a)$ is everywhere defined as a function of $a$; as a final corollary we note that in such cases, $\phi_{x_{0}}$ is everywhere defined. A similar comment holds with the words "everywhere defined" replaced by "primitive recursive."

Applications. We conclude with some comments on three applications of the Lemma.

Application 1. As a first and simplest application, consider the partial recursive function $\phi(a, b)=a+{ }_{o} b$ defined by Kleene in [3] by use of the recursion theorem. This function has the property 
(among others) that it is defined for $a, b \in O$ and that $\left|a+{ }_{o} b\right|$ $=|a|+|b|$ for all $a, b \in O$. (Recall that $O$ is a set of "notations" for ordinals; for $a \in O,|a|$ is the ordinal having $a$ as notation. $|a|+|b|$ indicates ordinary addition of ordinals.) The proof of the existence of a function with this property can be given, in a natural way, by use of the parametric form of the lemma. Take $a$ as parameter; and take $<_{W^{(a)}}$ to be $<_{o}$ if $a \in O$, and $W^{(a)}$ to be empty, if $a \notin O$. Let $D^{(a)}(x, S)$ assert: $\phi_{x}$ is defined on $S$ and gives an order preserving map of $S$ into $O$ such that for any $b \in S,\left|\phi_{x}(b)\right|=|a|+|b|$. Then conditions (i) and (ii) hold. $f^{(a)}$ is chosen to represent the procedure by which any such $\phi_{x}$ on $(b)^{(a)}$ can be extended to $(b]^{(a)}$. (If $b=1$, the new value at $b$ is $a$; if $b=2^{c}$, the new value at $b$ is $2^{\phi_{x}(c)}$; if $b=3 \cdot 5^{y}$, the new value at $b$ is $3 \cdot 5^{y^{\prime}}$ where $y^{\prime}$ is chosen so that $\phi_{y^{\prime}}=\phi_{x} \circ \phi_{y}$.) Then by the lemma, a recursive function $x_{0}(a)$ exists such that for $a \in O$, $\phi_{x_{0}(a)}$ gives an order preserving map of $O$ into $O$ with the property that $\left|\phi_{x_{0}(a)}(b)\right|=|a|+|b|$ for any $b \in O$. Hence, setting $\phi(a, b)$ $\sim \phi_{x_{0}(a)}(b)$, we have the desired partial recursive function.

Application 2. In 6.3 of [4], Kleene proves that there is a partial recursive function $\psi(a, b)$ such that if $a \leqq o b$ then $\psi(a, b)$ is an index for a procedure for $H_{a}$ recursive in $H_{b}$. (For terminology and notation see [6]. This result is also given as Theorem XIV in [6]). This proof falls under the scope of our lemma. We need only view $a$ as parameter, $W^{(a)}$ as $\{b \mid a \leqq o b\}, D^{(a)}(x, S)$ as the assertion: for every $b \in S$, $\phi_{x}(b)$ is defined and gives an index for $H_{a}$ recursive in $H_{b}$. The appropriate $f^{(a)}$ for condition (iii) follows from the definition of $H_{b}$ and the fact that for any $a, b \in O,(b)^{(a)}$ is recursively enumerable uniformly.

Application 3. Similarly, the final step in Spector's solution of the uniqueness problem for constructive ordinals, (Theorem 5 of [5]), involves an application of the recursion theorem that comes within the framework of the lemma. Our goal is to show that there is a partial recursive function $\gamma$ such that if $a, b \in O$ and $|a|=|b|$, then $\gamma(a, b)$ is an index for $H_{a}$ recursive in $H_{b}$. In this case we take $W$ $=\left\{2^{a} 3^{b} \mid a, b \in O\right.$ and $\left.|a|=|b|\right\}$, and $2^{a} 3^{b}<_{W} 2^{a^{\prime}} 3^{b^{\prime}}$ if $|a|<\left|a^{\prime}\right|$. $D(x, S)$ asserts: $\phi_{x}$ is defined on $S$ and, for $2^{a} 3^{b}$ in $S, \phi_{x}\left(2^{a} 3^{b}\right)$ gives an index for $H_{a}$ recursive in $H_{b}$. Spector has previously shown that $\{a|| a|=| b \mid\}$ is recursive uniformly in $H_{2}{ }^{2 b}$, and the existence of an appropriate $f$ for condition (iii) now follows straightforwardly from this fact and the constructions given by Spector. Hence the lemma applies (here in nonparametric form), and the desired $\gamma$ exists.

\section{REFERENCES}

1. S. Kleene, Introduction to metamathematics, New York, Van Nostrand, Amsterdam, North Holland, and Groningen, Noordhoff, 1952, $10+550$ pp. 
2. J. Myhill, Creative sets, Z. Math. Logik Grundlagen Math. vol. 1 (1955) pp. 97-108.

3. S. Kleene, On notation for ordinal numbers, J. Symb. Logic vol. 3 (1938) pp. 150-155.

4. - Arithmetical predicates and function quantifiers, Trans. Amer. Math. Soc. vol. 79 (1955) pp. 312-340.

5. C. Spector, Recursive well orderings, J. Symb. Logic, vol. 20 (1955) pp. 151163.

6. S. Kleene, Hierarchies of number theoretic predicates, Bull. Amer. Math. Soc. vol. 61 (1955) pp. 193-213.

7. E. Zermelo, Grundlagen einer allgemeinen Theorie der mathematischen Satzsysteme, Fund. Math. vol. 25 (1935) pp. 136-146.

Massachusetts Institute of Technology

\section{ON A SPECIAL INTEGRAL EQUATION ${ }^{1}$}

DAVID A. WOODWARD

1. Introduction. R. H. Cameron posed the following question ${ }^{2}$ in a paper [1]. Does

$$
y(t)=x(t)+\int_{0}^{t}[x(s)]^{2} d s, \quad 0 \leqq t \leqq 1,
$$

have a solution $x \in C$ for almost every choice of $y \in C$ ? Here $C$ denotes the space of continuous functions on $0 \leqq t \leqq 1$ which vanish at $t=0$, and "almost every" means all but a set of Wiener measure ${ }^{3}$ zero. The answer is no as we proceed to show.

2. We will show that if $y \in N=\{y \in C:|y(t)+4 t|<1 / 10,0 \leqq t \leqq 1\}$ then (1.1) has no solution $x$ among the elements of $C$. Then the answer to the question is no, since $N$, a uniform neighborhood, has positive measure.

Suppose that $y \in N, x \in C$, and (1.1) holds. Let

$$
Z(t)=\left\{\begin{array}{c}
0,0 \leqq t \leqq 1 / 10 \\
-4(t-1 / 10), 1 / 10 \leqq t \leqq \pi / 4+1 / 10
\end{array}\right.
$$

Received by the editors May 26, 1958 and, in revised form, March 7, 1959.

1 This research was supported by the United States Air Force, through the Office of Scientific Research of the Air Research and Development Command, under contract No. AF 18 (603)-30.

2 This question has two other formulations found in [1].

${ }^{3}$ See, for instance [2]. 